\title{
Telomerase activation in a model of lung adenocarcinoma
}

\author{
F. Suau*, V. Cottin*,\#, F. Archer*, S. Croze*, J. Chastang*, G. Cordier*, \\ F. Thivolet-Béjui*, ${ }^{\star}$, J-F. Mornex ${ }^{\star}, \#$ and C. Leroux*
}

ABSTRACT: Ovine pulmonary adenocarcinoma (OPA) is a lung cancer strikingly similar to the pneumonic-type mixed invasive adenocarcinoma with a predominant bronchioloalveolar component in humans. Telomerase activity in OPA and the potential involvement of the kinase Akt in telomerase activation and regulation of cell proliferation were investigated.

Lung tissues were collected from sheep with a histopathological diagnosis of OPA or controls. Epithelial cell cultures were derived in vitro from lung tissues. Telomerase activity was evaluated using the telomeric repeat amplification protocol method. Phosphorylation of Akt was detected by Western blotting.

Telomerase activity was significantly higher in OPA lung tissues compared to control lung tissues. A high telomerase activity was detected in eight out of 12 (67\%) primary cell cultures derived from tumours. A high level of expression of phosphorylated Akt was found in 10 out of 27 (37\%) tumours, with abolition of Akt activation in response to epidermal growth factor stimulation demonstrated in primary cell cultures derived from tumours.

Telomerase activation takes place in ovine pulmonary adenocarcinoma tumour cells and may be partly attributable to Akt activation. Telomerase may inhibit cellular senescence and contribute to the accumulation of tumour cells in mixed adenocarcinoma with a bronchioloalveolar component. Further work is necessary to identify alternative signalling pathways of telomerase activation in tumours.

KEYWORDS: Akt, bronchioloalveolar carcinoma, jaagsiekte sheep retrovirus, lung cancer, telomerase, type-II pneumocyte

ung cancer is the leading cause of cancer mortality in developed countries. Nonsmall cell lung cancer represents $\sim 80 \%$ of lung cancers, adenocarcinoma being the most frequent cell type, accounting for $\sim 40 \%$ of all cases of lung cancer. Lung adenocarcinoma may present as pneumonic-type adenocarcinoma (P-ADC), which associates typical radiological features with diffuse or disseminated alveolar condensation with or without an air bronchogram showing progression towards pulmonary right-to-left shunting, together with evidence of adenocarcinoma tumour cells in the lung [1]. The histological pattern in P-ADC may include bronchioloalveolar carcinoma (BAC), defined as an adenocarcinoma with a pure bronchioloalveolar growth pattern with no evidence of stromal, vascular or pleural invasion [2], or, more commonly, a mixed-type adenocarcinoma with a predominant bronchioloalveolar component and a papillary or acinar invasive component $[1,3]$.

For editorial comments see page 1079.
Ovine pulmonary adenocarcinoma (OPA) is a naturally occurring lung cancer that occurs spontaneously in sheep infected by the jaagsiekte sheep retrovirus (JSRV), and which may be reproduced by the experimental inoculation of lambs with the virus [4]. It grows with a disseminated pattern at the periphery of the lung. OPA is a mixed-type adenocarcinoma containing a significant proportion of a BAC component, together with papillary and acinar growth patterns $[2,3]$. OPA also shares striking clinical and radiological homology with human $\mathrm{P}-\mathrm{ADC}$, including progressive intrapulmonary spread and a lack of distant extrathoracic metastasis [3], and thus represents a unique natural and reproducible animal model of peripheral lung cancer, especially P-ADC.

In contrast with previous studies, which focused on defining the oncogenic properties of JSRV structural proteins in rodents [5], the aim of the present study was to identify pathogenic processes taking place in spontaneous tumours in vivo. Mechanisms potentially involved in tumour
AFFILIATIONS

*UMR754 Retroviruses and

Comparative Pathology INRA, University of Lyon I, National Veterinary School, EPHE, IFR128 Biosciences Lyon-Gerland, and Depts of ${ }^{\#}$ Respiratory Diseases, Reference Centre for Orphan Lung Diseases, and

"Pathology, Louis Pradel Hospital, Hospices Civils de Lyon, Lyon, France.

CORRESPONDENCE

V. Cottin

UMR754

Lyon Gerland

Université Lyon I

50 avenue Tony Garnier

69007 Lyon

France

Fax: 33437287605

E-mail: vincent.cottin@chu-lyon.fr

Received:

October 262005

Accepted after revision:

January 232006

\section{SUPPORT STATEMENT}

This study was supported by research grants from the Ligue nationale contre le cancer (Rhône, Drôme, Loire and Ardèche departmental committees) and the Région RhôneAlpes (Lyon, France). F. Suau is the recipient of a fellowship from the French Ministry of Research. 
formation include extensive cell division as a result of oncogenic mutations, and inactivation of cellular senescence, tumour suppressor pathways or apoptotic mechanisms that may otherwise arrest the proliferation or induce the death of potential cancer cells [6]. Cell senescence is a process mostly described in vitro, whereby primary normal cells grown in culture do not proliferate indefinitely but withdraw from the cell cycle (after a period of rapid proliferation) in response to diverse regulatory mechanisms including dysfunctional telomeres [7]. More recently, cellular senescence has been also demonstrated in vivo in pre-malignant but not malignant cells, suggesting that it may be an important anti-cancer defence [8].

Cellular senescence is mainly regulated by telomerase, a ribonucleoprotein enzyme able to stabilise telomere length by de novo synthesis of telomeres and elongation of existing telomeres. Telomerase activation is considered mandatory in order for tumour cells to escape cell senescence and gain increased proliferative capacities [9]. The telomerase reverse transcriptase (TERT) catalytic subunit is the major determinant of telomerase activity in vitro and in vivo. Activation of TERT has been well established in human cancer cell lines and tumours including lung cancer [10], whereas telomerase activity is repressed in most normal somatic cells. Complex regulation of telomerase activity may include the phosphatidylinositol-3'kinase (PI3K) pathway through phosphorylation (P) of TERT by Akt [11]. Involved in regulation of cell survival and cell cycle progression, Akt is constitutively activated in a variety of human tumours including lung cancer [12].

In the present study, increased telomerase activity was demonstrated in tumours and primary cultures of tumour cells derived from OPA, suggesting that inhibition of cell senescence may be involved in tumorigenesis and accumulation of tumoral cells within the lung. It was next shown that the regulatory kinase Akt is constitutively activated in OPA tumours and dysregulated in primary cultures derived from OPA, suggesting that Akt may be involved in telomerase activation in a proportion of tumours.

\section{MATERIALS AND METHODS \\ Lung tissues}

Lung tissues were collected immediately post mortem from sheep presenting with loss of weight, dypsnoea and profuse lung secretions suggestive of OPA or without signs of OPA. Tissue sections were sampled and stored at $-70^{\circ} \mathrm{C}$ until use or fixed in formalin for histopathological examination. The tissues were classified as tumoral and nontumoral lung (or controls) following the current 2004 World Health Organization classification [2].

\section{Culture of alveolar epithelial type II cells}

Tumour cells and normal type-II pneumocytes were isolated from ovine lungs and characterised. Briefly, tissue samples were digested overnight at $4{ }^{\circ} \mathrm{C}$ in Eagle's minimum essential medium (Eurobio, Courtaboeuf, France) containing 0.025\% collagenase I, $10 \mu \mathrm{g} \cdot \mathrm{mL}^{-1}$ deoxyribonuclease, $1 \mathrm{mg} \cdot \mathrm{mL}^{-1}$ protease XIV (Sigma-Aldrich, Saint-Quentin-Fallavier, France), $50 \mu \mathrm{g} \cdot \mathrm{mL}^{-1}$ streptomycin, $50 \mathrm{U} \cdot \mathrm{mL}^{-1}$ penicillin, $50 \mu \mathrm{g} \cdot \mathrm{mL}^{-1}$ gentamicin, $2.5 \mu \mathrm{g} \cdot \mathrm{mL}^{-1}$ amphotericin $\mathrm{B}$ and $100 \mathrm{U} \cdot \mathrm{mL}^{-1}$ nystatin. The cells were then homogenised and filtered through a $40-\mu \mathrm{m}$ filter in order to eliminate cell debris. After centrifugation for $10 \mathrm{~min}$ at $430 \times g$ at $4^{\circ} \mathrm{C}$, pulmonary cells were plated onto collagen, laminin and fibronectin-coated plates in selective medium for epithelial cells (Quantum 286; PAA Laboratories, Pasching, Austria) containing $5 \mathrm{ng} \cdot \mathrm{mL}^{-1}$ hepatocyte growth factor and $10 \mathrm{ng} \cdot \mathrm{mL}^{-1}$ keratinocyte growth factor (Abcys, Paris, France). Cells derived from OPA tumours $(n=12)$ and nontumoral lungs $(n=4)$ are referred to as tumoral and control cells, respectively.

\section{Detection of jaagsiekte sheep retrovirus proviral DNA}

Total genomic DNA from lung tumours and control lungs was prepared using the Fastprep device following the recommendations of the supplier (Qbiogene, Carlsbad, CA, USA). A semi-nested PCR protocol was used to detect proviral DNA. Briefly, reaction mixtures contained $500 \mathrm{ng}$ total genomic DNA, $50 \mu \mathrm{L} 1 \times$ buffer $\left(1.5 \mathrm{mM} \mathrm{MgCl}_{2}, 67 \mathrm{mM}\right.$ Tris- $\mathrm{HCl}$ (pH 8.8), $16 \mathrm{mM}\left(\mathrm{NH}_{4}\right)_{2} \mathrm{SO}_{4}, 0.01 \%$ Tween 20), $0.2 \mathrm{mM}$ each deoxynucleotide triphosphate, $0.2 \mathrm{mM}$ each primer and $1.25 \mathrm{U}$ Taq polymerase (Eurobio). The first round of PCR was performed with the primers JSRV42 (sense), 5'CTTTGTATTTCCCTGTGTCG-3', corresponding to nucleotides 7041-7060 in the env gene of the JSRV genomic sequence (GenBank accession number AF105220), and JSRV53 (antisense), 5'-GGATTCTTACACAATCACC-3', corresponding to nucleotides 7381-7362 in the long terminal repeat (LTR) U3 region of the JSRV genomic sequence. The second round of PCR was performed with 1-5 $\mu \mathrm{L}$ PCR product using JSRV42 and JSRV52 (antisense), 5'-CACCGGATTCTTATATAATC-3', corresponding to nucleotides 7366-7346 in the LTR U3 region of the JSRV genomic sequence. PCR reactions were performed as follows: $5 \mathrm{~min}$ at $95^{\circ} \mathrm{C}$, followed by 35 cycles of $30 \mathrm{~s}$ at $95^{\circ} \mathrm{C}$, $30 \mathrm{~s}$ at $50^{\circ} \mathrm{C}$ and $1 \mathrm{~min}$ at $72^{\circ} \mathrm{C}$, and with a final elongation of $10 \mathrm{~min}$ at $95^{\circ} \mathrm{C}$.

\section{Cell proliferation assay}

Cell numbers were determined using a 3-(4,5-dimethylthiazol2-yl)-2-5-diphenyl tetrazolium bromide (MTT) assay. Briefly, 5,000 cells $\cdot$ well $^{-1}$ were plated in 96 -well plates and incubated in a $5 \%$ carbon dioxide atmosphere for $96 \mathrm{~h}$ at $37^{\circ} \mathrm{C}$. MTT was incorporated as recommended for the MTT Cell Proliferation Assay kit (Chemicon, Temecula, CA, USA). MTT (10 $\mu \mathrm{L})$ was added to each well for $4 \mathrm{~h}$ at $37^{\circ} \mathrm{C}$. After solubilisation in $100 \mu \mathrm{L}$ $1 \mathrm{~N}$ isopropanol/0.04N HCl, absorbance was read at $595 \mathrm{~nm}$ using a Wallac Victor II device (Perkin-Elmer, Wellesley, MA, USA). Each measurement was performed in triplicate.

\section{Flow cytometry}

Quantitative measurement of the cell cycle was performed using flow cytometric analysis of nuclear DNA contents following propidium iodide staining. Briefly, $1 \times 10^{6}$ cells were harvested following dissociation with trypsin, washed twice in PBS and then fixed with $70 \%$ ethanol at $-20^{\circ} \mathrm{C}$. After one wash, cells were treated with $1 \mathrm{mg} \cdot \mathrm{mL}^{-1}$ ribonuclease A (SigmaAldrich) for $30 \mathrm{~min}$ at $4{ }^{\circ} \mathrm{C}$, incubated in $20 \mu \mathrm{g}$ propidium iodide (Sigma-Aldrich) and analysed by flow cytometry. The DNA content was analysed for $>10,000$ events using FACScan flow cytometry (Becton-Dickinson, Franklin Lakes, NJ, USA) with a 488-nm argon ion laser.

\section{Measurement of telomerase activity}

Frozen tissues or cells were lysed in $250 \mu$ ice-cold $1 \times 3$ ((3-cholamidopropyl)diethylammonio)-1-propane sulphonate 
(CHAPS) lysis buffer (Intergen Company, Purchase, NY, USA) and pulverised using the Fastprep device. The lysates were incubated on ice for $30 \mathrm{~min}$ and then centrifuged at $18,000 \times g$ for $30 \mathrm{~min}$ at $4^{\circ} \mathrm{C}$. The supernatants were collected and protein concentrations were measured with a modified Lowry protein assay (Perbio Science, Brébières, France). Telomerase activity was assayed using the telomeric repeat amplification protocol (TRAP) method using the TRAP-eze ELISA Telomerase Detection Kit (Intergen Company) following the manufacturer's recommendations. Briefly, $200 \mathrm{ng}$ total protein was used. Lysis buffer without protein was used as negative control. Control cell extracts containing telomerase activity and a synthetic oligonucleotide with eight telomeric repeats (supplied with the kit) were used as positive controls. For each cell sample, a heat-inactivated $\left(10 \mathrm{~min}\right.$ at $\left.85^{\circ} \mathrm{C}\right)$ negative control sample was also prepared. After amplification by PCR, the TRAP products were resolved on $12 \%$ polyacrylamide gels and visualised with 1:10,000 SYBR Gold (Molecular Probes (Invitrogen), Eugene, OR, USA). Amplification efficiency in each reaction was determined using the provided internal control oligonucleotides forming a 36-bp band. Telomerase activity was evaluated using a semi-quantitative ELISA method and expressed as the ratio of telomerase activity in the sample to telomerase activity in telomerase-positive control cells. All experiments were performed at least in duplicate. Measurements were reported as mean \pm SEM.

\section{Immunodetection of phosphorylated Akt, total Akt and capsid protein}

Frozen tissues or cells were lysed in $250 \mu \mathrm{L}$ lysis buffer $(0.5 \mathrm{M}$ Tris pH 8.0, 10\% glycerol, $150 \mathrm{mM} \mathrm{NaCl}, 1 \%$ Triton X-100, $5 \mathrm{mM}$ EDTA, $1 \mathrm{mM} \mathrm{Na} \mathrm{VO}_{4}, 1 \mathrm{mM}$ phenylmethylsulphonyl fluoride, $10 \mu \mathrm{g} \cdot \mathrm{mL}^{-1}$ leupeptin, $10 \mu \mathrm{g} \cdot \mathrm{mL}^{-1}$ aprotinin), homogenised with the Fastprep system device (Qbiogene) and incubated for $30 \mathrm{~min}$ on ice. The lysates were centrifuged at $18,000 \times g$ for $30 \mathrm{~min}$ at $4{ }^{\circ} \mathrm{C}$ and $50 \mu$ g protein were separated on a $12 \%$ sodium dodecylsulphate-polyacrylamide gel and transferred onto a $0.2-\mu \mathrm{M}$ nitrocellulose membrane (Biorad Laboratories, Marnes-la-Coquette, France). The membranes were pre-incubated with $25 \mathrm{mM}$ Tris ( $\mathrm{pH} 7.6) / 0.15 \mathrm{M} \mathrm{NaCl} /$ $0.05 \%$ Tween 20 (TSBT) containing 5\% non-fat dry milk for $1 \mathrm{~h}$ at room temperature $\left(20^{\circ} \mathrm{C}\right)$. After three washes in TSBT, the membranes were incubated overnight at $4^{\circ} \mathrm{C}$ with a $1: 1,000$ dilution of rabbit polyclonal antibodies directed against P-Akt (serine 473) or total Akt (Cell Signalling, Danvers, MA, USA) diluted in TSBT containing $5 \%$ bovine serum albumin. For detection of capsid antigenic protein, membranes were incubated for $1 \mathrm{~h}$ at room temperature with 1:10,000 diluted rabbit polyclonal antibody directed against JSRV capsid protein (generously provided by J.C. De Martini, Dept of Microbiology, Immunology and Pathology, Colorado State University, Fort Collins, CO, USA). Membranes were washed three times for $5 \mathrm{~min}$ at room temperature with TBST and incubated with horseradish peroxidase-labelled anti-rabbit immunoglobulin G (Sigma-Aldrich) diluted in TBST containing $5 \%$ non-fat dry milk $(1: 10,000)$ for $1 \mathrm{~h}$ at room temperature. The immunoreactive bands were detected using an enhanced chemiluminescence detection kit (Perbio Science), quantified with the Un-Scan-It software (Silk Scientific Corporation, Orem, UT, USA) and expressed as a percentage compared to A549 control cells. Measurements of expression of P-Akt were reported as mean \pm SEM. The protein quantity loaded on to gels was controlled by immunodetection of actin (Sigma-Aldrich) with a mouse monoclonal antibody directed against $\beta$-actin (Sigma-Adlrich).

\section{Statistical analysis}

Telomerase activity and P-Akt expression in tumoral and nontumoral samples were compared using the Mann-Whitney U-test. Tests were two-tailed unless otherwise detailed. Only $\mathrm{p}$-values of $<0.05$ were considered significant.

\section{RESULTS}

\section{Characterisation of tumours}

Lung tissue was obtained from OPA tumours and nontumoral control lungs. The diagnosis of OPA (invasive mixed adenocarcinoma with a bronchioloalveolar component, associated with acinar and/or papillary growth patterns) was confirmed by histopathological examination for all tumours, whereas absence of tumour was confirmed in all control lungs. In addition, lung tissues were assessed for the presence of the JSRV provirus and the capsid antigenic protein. JSRV proviral DNA was detected by PCR analysis in 11 out of 12 (92\%) tumour lungs and none of the four control lungs. Similarly, the JSRV capsid protein was detected by Western blot analysis in 12 out of 12 OPA lungs but none of the four control lungs.

\section{Telomerase activity in tumours}

As a first approach to studying the involvement of telomerase activation in OPA, telomerase activity was measured in lung tissues from OPA tumours $(n=12)$ and nontumoral control lungs $(n=4)$ using the TRAP assay. As illustrated in figure 1a, PCR products visualised as a DNA ladder on polyacrylamide gels indicated the presence of telomerase activity in all OPA samples $(n=12)$ but none of the samples from control lungs. Semi-quantification using ELISA demonstrated that the telomerase activity was significantly higher in OPA tissues compared to control lungs $(\mathrm{p}=0.03$ (one-tailed MannWhitney test); fig. 1b). These results thus demonstrate that high telomerase activity was present in the tumours (invasive mixed adenocarcinoma with a bronchioloalveolar component) in which JSRV infection was confirmed.

\section{Characterisation of tumoral cell cultures}

The next aim was to demonstrate that the telomerase activity observed in whole tumours was attributable to tumour cells per se (and not accompanying nontumoral cells present in the tumoral lung). Primary cultures were derived from tumours $(n=12)$ and control lungs $(n=4)$. These cells were characterised immunocytochemically using antibodies directed against specific markers of type-II pneumocytes (surfactant proteins A and C) and transmission electron microscopy. As expected, primary cells derived from tumours and normal lungs showed the cuboidal morphology typical of epithelial cells. The purity of the cultures was confirmed by expression of surfactant proteins $\mathrm{A}$ and $\mathrm{C}$ in $>95 \%$ of the cells, and was maintained over all passages. The JSRV genome was detected by PCR analysis in cell cultures derived from all OPA lungs, but in none of the control type-II pneumocytes derived from control lungs. Cultured cells derived from tumours could be maintained for seven to ten passages, as compared to only two or three passages for control cells. 
a)
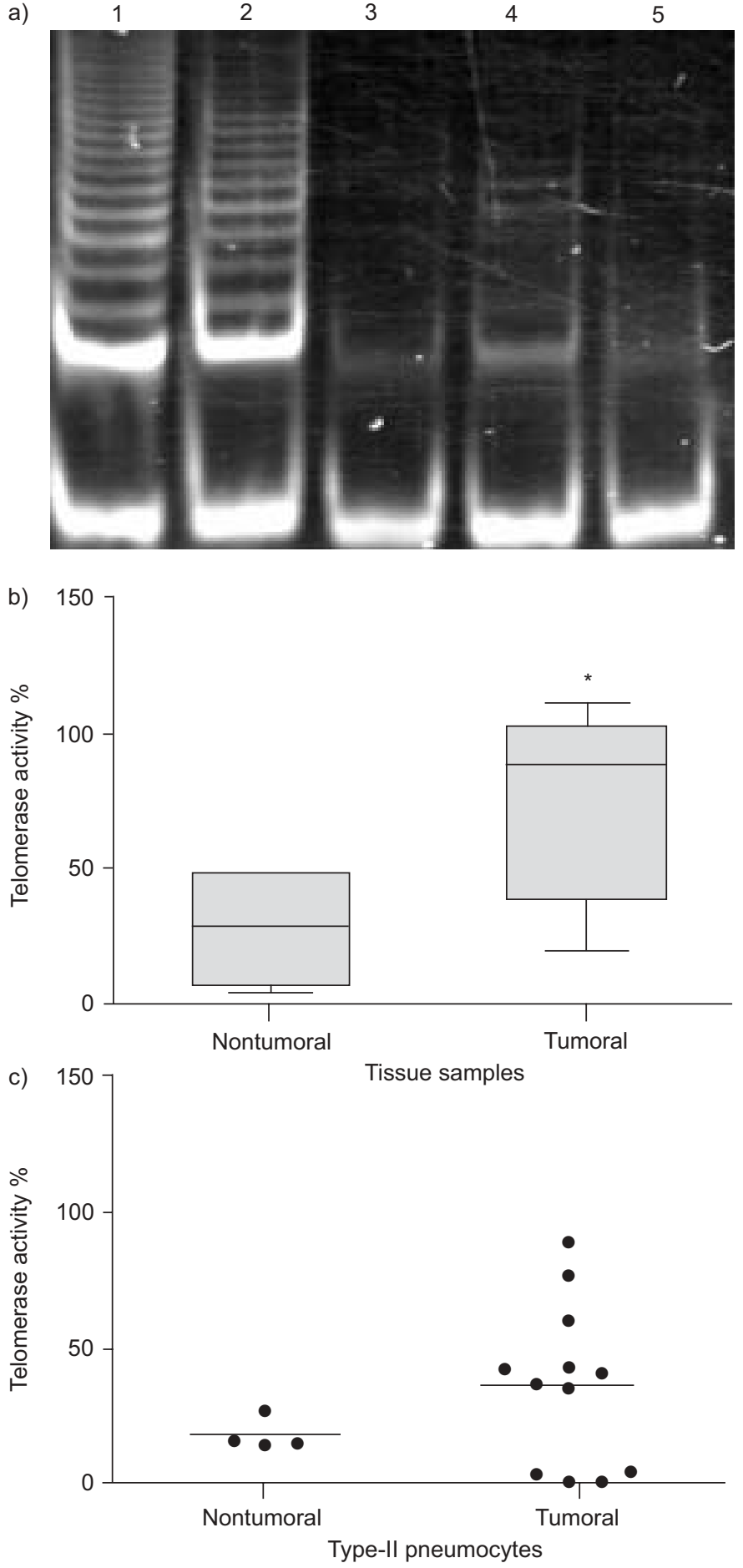

FIGURE 1. a) Polyacrylamide gel analysis of PCR products of the telomeric repeat amplification protocol assay in lung tumours (lanes 2 and 3 ) and control lung tissues (lanes 4 and 5). Heat-inactivated samples (lanes 3 and 5) were used as negative controls, and a telomerase-positive control cell line as a positive control (lane 1). Telomerase activity in: b) ovine pulmonary adenocarcinoma $(n=12)$ and control lung tissues $(n=4)$; and c) cell cultures (type-ll pneumocytes) derived from tumours $(n=12)$ and control lungs $(n=4)$. Boxes represent median and vertical bars represent interquartile range. Horizontal bars represent medians *: $p<0.05$.
In order to assess the biological relevance of cell cultures derived from tumours and control lungs, cell proliferation and distribution were analysed throughout the cell cycle of tumour and control cells. Using an MTT assay, it was shown that cells derived from lung tumours exhibited a significant proliferative advantage compared to control type-II pneumocytes derived from control lungs (fig. 2). Similarly, cell cycle analysis using flow cytometry indicated that the proportion of cells in the $S$ phase was higher in cultures of tumoral cells $(17 \pm 3.2 \%)$ than in control type-II pneumocytes $(8.5 \pm 1.9 \%)$.

\section{Telomerase activity in tumoral cell cultures}

In order to demonstrate that the telomerase activity observed in whole tumours was attributable to tumour cells per se, telomerase activity was assessed in primary cell cultures derived from tumours and controls. Semi-quantitative measurement of telomerase activity using the TRAP assay followed by an ELISA demonstrated a particularly high level of telomerase activity compared to control type-II pneumocytes, in which a low level of enzymatic activity could be detected, in eight out of $12(67 \%)$ primary cultures derived from tumours (fig. 1c). Surprisingly, no telomerase activity was found in four out of $12(33 \%)$ cell cultures derived from tumours (in which telomerase activity had been detected in the whole tumour). Therefore, these results show that a high level of telomerase activity was present in the majority of cell cultures derived from lung tumours, and indicated that the telomerase activity observed in whole tumours was indeed attributable to tumour cells per se in the majority of tumours.

\section{Dysregulation of Akt in tumoral cell cultures}

Regulation of telomerase activity is complex and may involve activation of TERT through its phosphorylation by the kinase Akt. In order to determine whether telomerase activation might be related to the Akt pathway, the phosphorylation state of serine 473 of Akt was studied in OPA-derived cell cultures. Type-II pneumocytes derived from tumours and normal lungs were cultured in the absence of growth factors for $24 \mathrm{~h}$, and

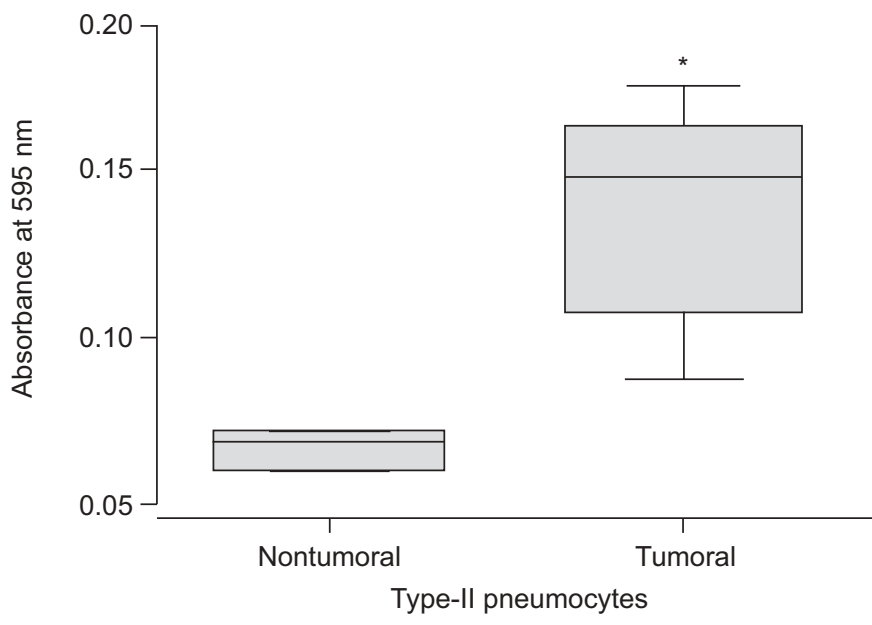

FIGURE 2. Cell proliferation of cultures derived from ovine pulmonary adenocarcinoma tumours and normal lungs (3-(4,5-dimethylthiazol-2-yl)-2-5-diphenyl tetrazolium bromide assay). Boxes represent median and interquartile range, with vertical bars representing range. *: $p<0.05$. 
then exposed or not to $100 \mathrm{ng} \cdot \mathrm{mL}^{-1}$ epidermal growth factor (EGF), a known activator of Akt in mammalian cells, for $30 \mathrm{~min}$. Similar moderate expression of P-Akt was detected by Western blotting in unstimulated cells derived from lung tumours and control type-II pneumocytes (fig. 3), suggesting that the culture conditions (including deprivation of growth factors) might lead to basal phosphorylation and activation of Akt in unstimulated cells. However, EGF stimulation in vitro led to a dramatic increase in Akt activation in control cells, whereas cells derived from lung tumours were not responsive to stimulation by EGF (fig. 3). These results showed that the EGF-Akt pathway was dysregulated in OPA cell cultures, with lack of Akt activation in response to growth factor stimulation in tumoral cell cultures.

\section{Activation of Akt in tumours}

In order to further characterise the dysregulation of Akt in OPA, Akt activation was next studied in whole tumours $(n=27)$ and normal lung tissues $(n=14)$ using Western blotting analysis, with human A549 epithelial cells as a reference. As shown in figure 4, significant expression of P-Akt was
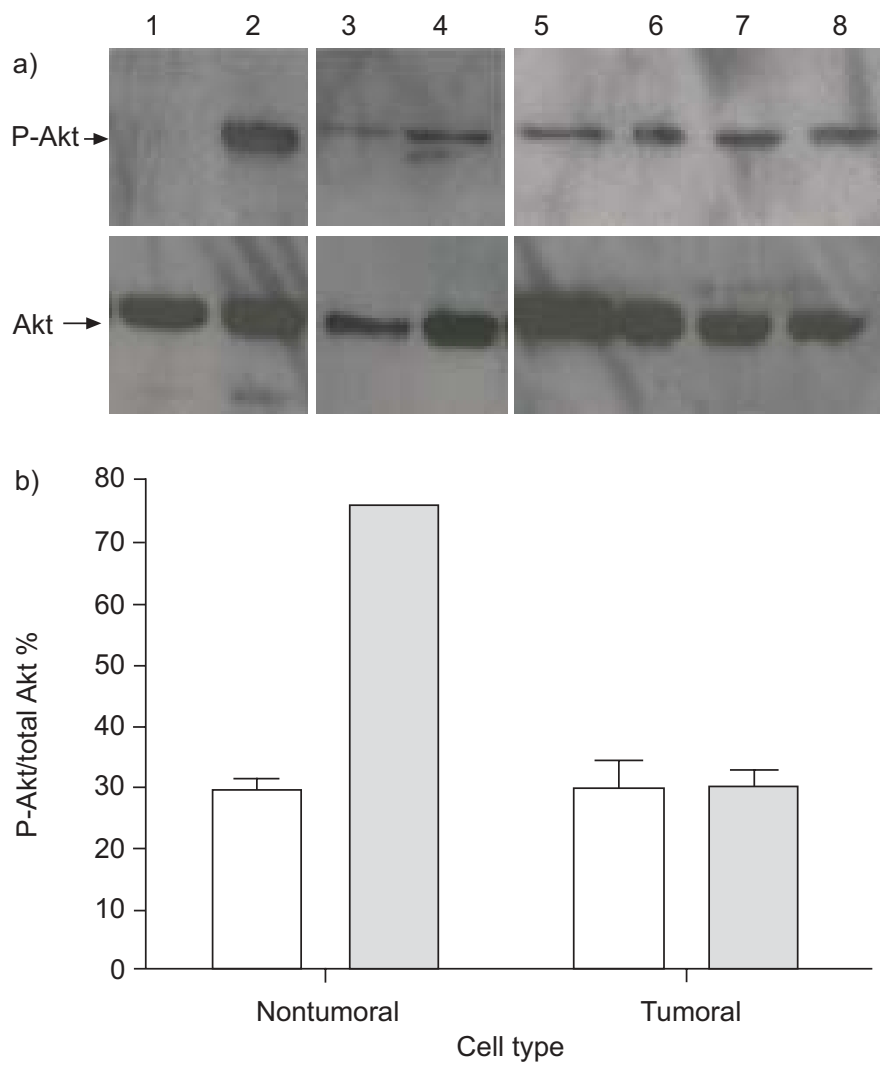

FIGURE 3. Dysregulation of Akt in cells derived from ovine pulmonary adenocarcinoma (OPA) tumours. Cells were deprived of serum and growth factors for $24 \mathrm{~h}$ and then exposed (lanes 2, 4, 6 and 8; $\square$ ) or not (lanes 1, 3, 5 and 7; $\square$ ) to stimulation with $100 \mathrm{ng} \cdot \mathrm{mL}^{-1}$ epidermal growth factor (EGF) for $30 \mathrm{~min}$. a) Western blot analysis of phosphorylated (P)-Akt (serine 473) in cell lysates from cultures derived from OPA lung tumours (lanes 5-8), A549 control cells (lanes 1 and 2) and normal lungs (lanes 3 and 4). The amount of total Akt was determined by re-probing the membranes with an antibody directed against Akt. b) Densitometric quantification of P-Akt and total Akt detected by Western blot analysis. Data are presented as mean \pm SEM. demonstrated in 10 of 27 (37\%) OPA tumours and none of 14 control lungs (table 1), whereas expression of total Akt and actin were similar between the two groups. Thus Akt is activated and may thus participate in telomerase activation in a significant proportion of OPA tumours.

\section{DISCUSSION}

In the present study, it was shown that telomerase is activated in OPA, a mixed-type adenocarcinoma of the lung with a prominent bronchioloalveolar component. Telomerase activation was demonstrated in tumours as well as in epithelial cell cultures derived from those tumours. Next the activation of the regulatory kinase Akt, as a potential activator of telomerase, was investigated, and it was demonstrated that Akt is indeed activated in a proportion of tumours. Taken together, these results suggest that inhibition of cell senescence may be involved in tumorigenesis and accumulation of tumoral cells within the lung in OPA, and that Akt activation may participate in telomerase activation in a proportion of tumours.

P-ADC differs from other types of nonsmall cell lung cancer in several ways, including a higher incidence in females, lesser role of tobacco smoking, lack of distant metastatic spread and higher rate of sensitivity to EGF tyrosine kinase inhibitors. The clinical syndrome of P-ADC remains a rare presentation of nonsmall cell lung cancer, hampering research.
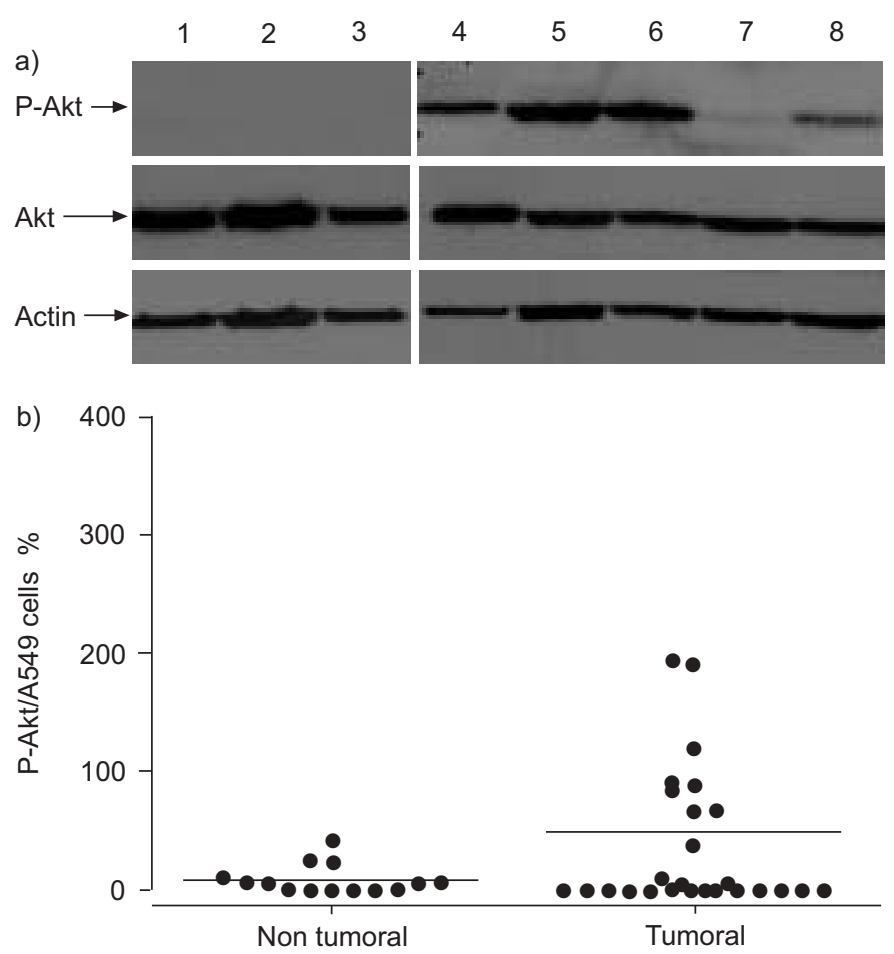

FIGURE 4. Activation of Akt in ovine pulmonary adenocarcinoma tumours a) Western blot analysis of phosphorylated (P)-Akt (serine 473), total Akt and $\beta$-actin in lysates of tumoral (lanes 5-8) and control lung tissues (lanes 1-3) and A549 control cells (lane 4). b) Densitometric quantification of P-Akt in sample (tumoral $(n=27)$ and nontumoral $(n=14))$ relative to reference epidermal growth factorstimulated A549 cells. Points represent means of two or three independent experiments; horizontal bars indicate medians. 


\begin{tabular}{lcc} 
TABLE 1 & $\begin{array}{l}\text { Activation (phosphorylation }(P)) \text { of } \text { Akt }^{\#} \\
\text { pulmonary adenocarcinoma tumours }\end{array}$ \\
\hline P-Akt & Nontumoral lung & Tumours \\
\hline- & $10(71.4)$ & $16(59.3)$ \\
+ & $4(28.6)$ & $1(3.7)$ \\
++ & $0(0)$ & $10(37)$ \\
\hline
\end{tabular}

Data are presented as n (\%). -: 0-9\%; +: 10-49\%; ++: $>50 \%$ of P-Akt level in A549 cells. ${ }^{*}$ : P-Akt was detected by Western blotting (whole tumours $(n=27)$ and normal lung tissues $(n=14)$ ), quantified densitometrically and expressed relative to P-Akt levels in human A549 epithelial cells.

OPA was chosen as a model of P-ADC since it shares with the human disease a variety of key features, including similar clinical and radiological presentation with progressive dyspnoea, and abundant bronchorrhoea; multifocal pulmonary disease with alveolar consolidation and nodules; and almost identical pathology [3]. As in BAC, tumour cells in OPA derive from type-II pneumocytes and, to a lesser extent, Clara cells. The BAC component of both the human and the ovine tumours is characterised by lepidic spread, whereby tumoral cells grow following the alveolar septa; as opposed to the rare form of pure BAC, mixed adenocarcinoma with bronchioloalveolar component includes evidence of stromal, vascular and/or pleural invasion, together with associated acinar and mostly papillary growth patterns $[1,3]$. There is evidence that OPA is immunohistochemically similar to human mixed BAC [13], with expression of cytokeratin 7, nuclear expression of thyroid transcription factor-1 and lack of expression of cytokeratin 20, and by electronic microscopic studies (data not shown). As opposed to previous work on OPA, in which studies were mostly conducted in immortalised cell lines or rodent fibroblasts transfected with JSRV gene expression vectors [14-16], in the present study, it was possible to derive primary cell cultures from naturally occurring tumours and control lungs. Genomic proviral DNA of the causative agent JSRV was detected only in cells derived from tumours. In addition, cells derived from OPA tumours expressed surfactant pulmonaryassociated proteins A and C (markers of type-II pneumocytes) and thyroid transcription factor-1, indicating that their specific phenotype was maintained in vitro. Importantly, concordant results of telomerase activation were obtained in parallel in tumours and epithelial cell cultures derived from the tumours, doubtlessly increasing the biological relevance of the present observation.

A high level of telomerase activity was demonstrated in OPA lung tumours compared to control nontumoral lungs. Telomerase activity was detected in all OPA tumours, and was probably due to telomerase activation within tumour cells. As cells located within the tumour other than cancer cells per se [17-19], such as lymphocytes of the bronchial mucosa [20], may also express telomerase activity, primary cell cultures were derived from the same lung tumours; telomerase activity was found in two-thirds of epithelial cell cultures derived from tumours, thus indicating that the telomerase activity observed in whole tumours was at least partly attributable to tumour cells per se. No telomerase activity was found in a third of cell cultures derived from telomerase-positive tumours; the present authors hypothesise that activated lymphocytes or other inflammatory cells infiltrating the tumour may have been responsible for telomerase activity in such cases, as previously reported [17, 18]. It remains to be determined whether alternative pathways to telomerase activation may also contribute to escaping cell senescence in telomerase-negative tumour cell cultures [21]. Conversely, low telomerase activity was found in control lung tissues and cell cultures, potentially resulting from a subpopulation of type-II pneumocytes with self-renewal capacities that are assumed to repair lung alveolar epithelium after injury [22]. Alternatively, telomerase activity in control lungs may be due to activated lymphocytes within nontumoral lungs as a result of a variety of infraclinical infections that are common in naturally bred animals, although this was not suggested by the pathological analysis of control lungs in the present study.

Telomerase activation in OPA suggests that inhibition of cell senescence may be involved in tumorigenesis, and in the process of accumulation of tumour cells along the alveolar septa. Telomeres terminate eukaryotic chromosomes and are involved in chromosomal integrity. Continued telomere shortening in normal somatic cells eventually results in an arrest of cell proliferation, a physiological process referred to as cell senescence, which controls cell lifespan and limits the number of cell divisions [9]. Activation of telomerase activity, mainly dependent on its catalytic subunit TERT, contributes to telomere length maintenance and inhibition of cell senescence, and thus to cell immortalisation and cancer [9]. Hence, maintained telomere length was found in OPA (data not shown). In addition to its enzymatic activity, the TERT subunit may enhance genomic stability by direct interaction with telomeres [23], and may participate in the regulation of p53induced apoptosis [24]. Telomerase activation is an early event in carcinogenesis, concomitant with p53 overexpression, retinoblastoma protein $(\mathrm{Rb})$ inactivation and a decrease in B-cell leukaemia/lymphoma 2 gene product (Bcl-2)/Bcl-2associated $X$ protein (Bax) ratio in high-grade pre-invasive bronchial lesions (beginning at the level of moderate dysplasia), suggesting a coupling between telomerase activation, proliferation and resistance to apoptosis [20]. Although telomerase activation has been described in a variety of human cancers, including lung cancer [10], it has not been extensively studied in P-ADC and mixed adenocarcinoma with BAC features. Some telomerase activity, as assessed by a nonquantitative method, was detected in four of 10 cases of human BAC presenting as solitary nodules [25], and in $97 \%$ of peripheral and small-sized nonmucinous BAC [26], but such tumours were probably not representative of the clinically defined P-ADC [1]. Interestingly, telomerase activation alone is not sufficient to transform human cells in vitro [27]. Mechanisms other than cell senescence inhibition (such as inhibition of apoptosis, or deregulation of cell proliferation) may also take place in P-ADC and OPA, as suggested by the increased proliferation of tumour-derived cell cultures that was observed compared to control cells.

The complex regulation of telomerase activity involves several pathways, including the phosphorylation and activation of TERT by Akt [11], a kinase involved in the regulation of processes characteristic of cancer, such as cell proliferation and 
survival, cell size, response to nutrient availability, angiogenesis and tissue invasion [28]. Overexpression of Akt can transform NIH3T3 cells, indicating that Akt is a potential oncogene [29]. The potential role of Akt in telomerase activation was studied, and dysregulation of the EGF-Akt pathway observed in OPA tumours. Hence, Akt activation was present in a significant proportion of OPA tumours but none of the control lungs. Lack of Akt activation in response to EGF stimulation was further demonstrated in cell cultures derived from tumours, as compared to control cells (in which EGF stimulation dramatically induced Akt phosphorylation), demonstrating that the EGF-Akt pathway is dysregulated in cells derived from tumours. The basal level of Akt activation was comparable in cell cultures derived from tumours and nontumoral lung, a finding probably related to artefacts of cell culture (deprivation of growth factors or replacement of medium may have contributed to moderate activation of Akt in both tumoral and nontumoral cell cultures [28]). Taken together, the present results suggest that Akt activation may participate in telomerase activation and regulation of cell senescence in OPA, as recently shown in human mixed adenocarcinoma with BAC features [30]. Alternative pathways such as the Ras-mitogen-activated protein kinase kinase (MEK)-mitogen-activated protein kinase (MAPK) pathway [15] are also likely to participate in telomerase regulation in this tumour, as Akt activation was not found in all telomerasepositive tumours.

Several studies have identified the gene encoding the envelope of JSRV (env), the causative agent of OPA, as a potential oncogene, and have shown that its overexpression is sufficient to transform rodent fibroblasts [31] and epithelial cell lines in vitro [14]. Transfection of JSRV env in mammalian cells induces constitutive activation of Akt; studies using chemical inhibitors of the PI3K-Akt-mammalian-target-of-rapamycin pathway have further demonstrated the central role of Akt and of the Ras-MEK-MAPK pathway, in JSRV-induced cell transformation [15, 32], although the precise mechanism of Akt activation in naturally occurring OPA remains speculative.

Recently, activating mutations of the EGF receptor (EGFR) mutually exclusive of $\mathrm{K}$-ras mutations have been reported in lung adenocarcinoma, especially with a prominent nonmucinous BAC component, and may be associated with clinical response to the EGFR tyrosine kinase inhibitors gefitinib and erlotinib [33-35]. Activation of EGFR is associated with activation of downstream signalling pathways, including the PI3K-Akt and the Ras-MEK-extracellular-signal-regulatedkinase pathways in this tumour $[30,36]$. In addition, increased Akt phosphorylation (not inhibited by EGFR tyrosine kinase inhibitors) has been demonstrated in vitro in specific subpopulations of adenocarcinoma cell lines that have become naturally resistant to gefitinib, despite the loss of the EGFR gene mutation compared to parental cell lines [37]. These observations thus indicate that constitutive activation of the PI3K-Akt pathway may occur independently of EGFR mutations, and may be an attractive therapeutic target in lung adenocarcinoma. Studies are currently being undertaken in order to determine whether dysregulation of the EGF-Akt pathway in cells derived from OPA may be similarly related to mutations of the EGFR gene.
In conclusion, it has been shown that telomerase activation takes place in ovine pulmonary adenocarcinoma tumour cells, and may be partly attributable to Akt activation. Telomerase activation may contribute to the accumulation of tumour cells within the lung through inhibition of cellular senescence. Future strategies for the treatment of human pneumonic-type adenocarcinoma with bronchioloalveolar carcinoma features may be envisioned, through telomerase specific inhibition and/or modulation of the Akt pathway. Ovine pulmonary adenocarcinoma provides an attractive model for the preclinical assessment of the efficacy of innovative approaches to treat this incurable disease.

\section{REFERENCES}

1 Wislez M, Massiani MA, Milleron B, et al. Clinical characteristics of pneumonic-type adenocarcinoma of the lung. Chest 2003; 123: 1868-1877.

2 Travis WD, Brambilla E, Muller-Hemerlink HK, Harris CC. World Health Organization Classification of Tumours. Pathology and Genetics of Tumours of the Lung, Pleura, Thymus and Heart. Lyon, International Agency for Research on Cancer Press, 2004.

3 Mornex JF, Thivolet F, De las Heras M, Leroux C. Pathology of human bronchioloalveolar carcinoma and its relationship to the ovine disease. Curr Top Microbiol Immunol 2003; 275: 225-248.

4 Palmarini M, Sharp JM, de las Heras M, Fan H. Jaagsiekte sheep retrovirus is necessary and sufficient to induce a contagious lung cancer in sheep. J Virol 1999; 73: 6964-6972.

5 Wootton SK, Halbert CL, Miller AD. Sheep retrovirus structural protein induces lung tumours. Nature 2005; 434: 904-907.

6 Hanahan D, Weinberg RA. The hallmarks of cancer. Cell 2000; 100: 57-70.

7 Sharpless NE, DePinho RA. Telomeres, stem cells, senescence, and cancer. J Clin Invest 2004; 113: 160-168.

8 Collado M, Gil J, Efeyan A, et al. Tumour biology: senescence in premalignant tumours. Nature 2005; 436: 642.

9 Mathon NF, Lloyd AC. Cell senescence and cancer. Nat Rev Cancer 2001; 1: 203-213.

10 Lee JC, Jong HS, Yoo CG, Han SK, Shim YS, Kim YW. Telomerase activity in lung cancer cell lines and tissues. Lung Cancer 1998; 21: 99-103.

11 Kang SS, Kwon T, Kwon DY, Do SI. Akt protein kinase enhances human telomerase activity through phosphorylation of telomerase reverse transcriptase subunit. J Biol Chem 1999; 274: 13085-13090.

12 Shah A, Swain WA, Richardson D, et al. Phospho-Akt expression is associated with a favorable outcome in nonsmall cell lung cancer. Clin Cancer Res 2005; 11: 2930-2936.

13 Simsir A, Wei XJ, Yee H, Moreira A, Cangiarella J. Differential expression of cytokeratins 7 and 20 and thyroid transcription factor-1 in bronchioloalveolar carcinoma: an immunohistochemical study in fine-needle aspiration biopsy specimens. Am J Clin Pathol 2004; 121: 350-357.

14 Danilkovitch-Miagkova A, Duh FM, Kuzmin I, et al. Hyaluronidase 2 negatively regulates $\mathrm{RON}$ receptor tyrosine kinase and mediates transformation of epithelial 
cells by jaagsiekte sheep retrovirus. Proc Natl Acad Sci USA 2003; 100: 4580-4585.

15 Maeda N, Fu W, Ortin A, de las Heras M, Fan H. Roles of the Ras-MEK-mitogen-activated protein kinase and phosphatidylinositol 3-kinase-Akt-mTOR pathways in jaagsiekte sheep retrovirus-induced transformation of rodent fibroblast and epithelial cell lines. J Virol 2005; 79: 4440-4450.

16 Liu SL, Duh FM, Lerman MI, Miller AD. Role of virus receptor Hyal2 in oncogenic transformation of rodent fibroblasts by sheep betaretrovirus Env proteins. J Virol 2003; 77: 2850-2858.

17 Onishi T, Nouso K, Higashi T, et al. Cellular distribution of telomerase reverse transcriptase in human hepatocellular carcinoma. J Gastroenterol Hepatol 2003; 18: 1168-1174.

18 Fukushima M, Shimomura N, Nakamura K, et al. Demonstration of human telomerase reverse transcriptase by in situ hybridization in lung carcinoma. Oncol Rep 2004; 12: 1227-1232.

19 Lantuejoul S, Soria JC, Moro-Sibilot D, et al. Differential expression of telomerase reverse transcriptase (hTERT) in lung tumours. Br J Cancer 2004; 90: 1222-1229.

20 Lantuejoul S, Soria JC, Morat L, et al. Telomere shortening and telomerase reverse transcriptase expression in preinvasive bronchial lesions. Clin Cancer Res 2005; 11: 2074-2082.

21 Bryan TM, Englezou A, Dalla-Pozza L, Dunham MA, Reddel RR. Evidence for an alternative mechanism for maintaining telomere length in human tumors and tumorderived cell lines. Nat Med 1997; 3: 1271-1274.

22 Driscoll B, Buckley S, Bui KC, Anderson KD, Warburton D. Telomerase in alveolar epithelial development and repair. Am J Physiol Lung Cell Mol Physiol 2000; 279: L1191-L1198.

23 Sharma GG, Gupta A, Wang H, et al. hTERT associates with human telomeres and enhances genomic stability and DNA repair. Oncogene 2003; 22: 131-146.

24 Rahman R, Latonen L, Wiman KG. hTERT antagonizes p53-induced apoptosis independently of telomerase activity. Oncogene 2005; 24: 1320-1327.

25 Marchetti A, Bertacca G, Buttitta F, et al. Telomerase activity as a prognostic indicator in stage I non-small cell lung cancer. Clin Cancer Res 1999; 5: 2077-2081.

26 Nakanishi K, Kawai T, Kumaki F, Hirot S, Mukai M, Ikeda E. Expression of human telomerase RNA component and telomerase reverse transcriptase mRNA in atypical adenomatous hyperplasia of the lung. Hum Pathol 2002; 33: 697-702.

27 Morales CP, Holt SE, Ouellette M, et al. Absence of cancerassociated changes in human fibroblasts immortalized with telomerase. Nat Genet 1999; 21: 115-118.

28 Bellacosa A, Kumar CC, Di Cristofano A, Testa JR. Activation of AKT kinases in cancer: implications for therapeutic targeting. Adv Cancer Res 2005; 94: 29-86.

29 Cheng JQ, Altomare DA, Klein MA, et al. Transforming activity and mitosis-related expression of the AKT2 oncogene: evidence suggesting a link between cell cycle regulation and oncogenesis. Oncogene 1997; 14: 2793-2801.

30 Erman M, Grunenwald D, Penault-Llorca F, et al. Epidermal growth factor receptor, HER-2/neu and related pathways in lung adenocarcinomas with bronchioloalveolar features. Lung Cancer 2005; 47: 315-323.

31 Maeda N, Palmarini M, Murgia C, Fan H. Direct transformation of rodent fibroblasts by jaagsiekte sheep retrovirus DNA. Proc Natl Acad Sci USA 2001; 98: 4449-4454.

32 Zavala G, Pretto C, Chow YH, et al. Relevance of Akt phosphorylation in cell transformation induced by Jaagsiekte sheep retrovirus. Virology 2003; 312: 95-105.

33 Lynch TJ, Bell DW, Sordella R, et al. Activating mutations in the epidermal growth factor receptor underlying responsiveness of non-small-cell lung cancer to gefitinib. N Engl J Med 2004; 350: 2129-2139.

34 Paez JG, Janne PA, Lee JC, et al. EGFR mutations in lung cancer: correlation with clinical response to gefitinib therapy. Science 2004; 304: 1497-1500.

35 Miller VA, Kris MG, Shah N, et al. Bronchioloalveolar pathologic subtype and smoking history predict sensitivity to gefitinib in advanced non-small-cell lung cancer. J Clin Oncol 2004; 22: 1103-1109.

36 Yoshida Y, Shibata T, Kokubu A, et al. Mutations of the epidermal growth factor receptor gene in atypical adenomatous hyperplasia and bronchioloalveolar carcinoma of the lung. Lung Cancer 2005; 50: 1-8.

37 Kokubo Y, Gemma A, Noro R, et al. Reduction of PTEN protein and loss of epidermal growth factor receptor gene mutation in lung cancer with natural resistance to gefitinib (IRESSA). Br J Cancer 2005; 92: 1711-1719. 